ARTICLE OPEN

\title{
Hippocampal place cell and inhibitory neuron activity in disrupted-in-schizophrenia-1 mutant mice: implications for working memory deficits
}

\author{
Lia Mesbah-Oskui ${ }^{1,2}$, John Georgiou ${ }^{2}$ and John C Roder ${ }^{1,2,3}$
}

BACKGROUND: Despite the prevalence of working memory deficits in schizophrenia, the neuronal mechanisms mediating these deficits are not fully understood. Importantly, deficits in spatial working memory are identified in numerous mouse models that exhibit schizophrenia-like endophenotypes. The hippocampus is one of the major brain regions that actively encodes spatial location, possessing pyramidal neurons, commonly referred to as 'place cells', that fire in a location-specific manner. This study tests the hypothesis that mice with a schizophrenia-like endophenotype exhibit impaired encoding of spatial location in the hippocampus.

AIMS: To characterize hippocampal place cell activity in mice that exhibit a schizophrenia-like endophenotype.

METHODS: We recorded CA1 place cell activity in six control mice and six mice that carry a point mutation in the disrupted-inschizophrenia-1 gene (Disc1-L100P) and have previously been shown to exhibit deficits in spatial working memory.

RESULTS: The spatial specificity and stability of Disc1-L100P place cells were similar to wild-type place cells. Importantly, however, Disc1-L100P place cells exhibited a higher propensity to increase their firing rate in a single, large location of the environment, rather than multiple smaller locations, indicating a generalization in their spatial selectivity. Alterations in the signaling and numbers of CA1 putative inhibitory interneurons and decreased hippocampal theta $(5-12 \mathrm{~Hz})$ power were also identified in the Disc1-L100P mice.

CONCLUSIONS: The generalized spatial selectivity of Disc1-L100P place cells suggests a simplification of the ensemble place codes that encode individual locations and subserve spatial working memory. Moreover, these results suggest that deficient working memory in schizophrenia results from an impaired ability to uniquely code the individual components of a memory sequence.

npj Schizophrenia (2015) 1, Article number: 15011; doi:10.1038/npjschz.2015.11; published online 1 April 2015

\section{INTRODUCTION}

Impaired working memory is the most widely identified cognitive symptom in patients with schizophrenia ${ }^{1-3}$ and the severity of these deficits serve as one of the best predictors of patient outcome. ${ }^{4,5}$ While the exact mechanisms mediating working memory are not understood, it is a system that executes the transient recall of memories to serve immediate future action. ${ }^{6}$ Thus, an impaired ability to initially encode an event in memory may contribute to deficiencies in working memory.

Mutations in the disrupted-in-schizophrenia-1 (Disc1) gene have been associated with schizophrenia in a number of studies and numerous Disc1 mouse strains exhibit schizophrenia-like endophenotypes. ${ }^{7-13}$ These mouse models incorporate face validity with respect to deficits in spatial working memory and provide a powerful model system that can be used to understand the mechanisms underlying symptoms that may be relevant for schizophrenia and mental illness. ${ }^{7-10}$ Mechanistically, hippocampal place cells are one of the major systems involved in the encoding of spatial location, with place cells exhibiting location-specific firing patterns that are unique for each environment. ${ }^{14}$ Ensembles of simultaneously active place cells can serve to encode distinct environmental locations, with each location possessing a different complexion of active place cells. ${ }^{15,16}$
We monitored hippocampal place cell activity in freely behaving Disc1-L100P and control mice to identify any alterations in the spatial specificity and/or selectivity of Disc1-L100P place cells, which could contribute to the deficits in spatial working memory previously identified in these mice. ${ }^{4}$ Identifying an in vivo functional readout of place cell dysfunction would have broad implications for impaired working memory in schizophrenia and other disorders. We also examined hippocampal theta $(5-12 \mathrm{~Hz})$ power and the activity and numbers of putative inhibitory interneurons, as both decreased theta power and aberrant inhibitory signaling are associated with schizophrenia and impaired working memory. ${ }^{17,18}$

Here we show that Disc1-L100P mice exhibit faithful place cell activity, firing in a spatially restricted manner that results in welldefined fields of increased activity (i.e., place fields). Disc1-L100P place cells, however, show increased firing in a single, large location, compared with wild-type place cells that increase their firing rates in multiple locations, indicating a generalization in the spatial selectivity of Disc1-L100P place cells. Disc1-L100P mice also exhibit a deficit in hippocampal theta power, altered putative inhibitory interneuron action potential kinetics, and alterations in the distribution of CA1 parvalbumin-positive interneurons that lead to fewer parvalbumin-positive cells in the central hippocampus (i.e., -1.5 to $-2.5 \mathrm{~mm}$ from Bregma). Together, these findings indicate that the ability to register spatial location is intact in

\footnotetext{
${ }^{1}$ Department of Physiology, University of Toronto, Toronto, ON, Canada; ${ }^{2}$ Lunenfeld-Tanenbaum Research Institute, Mount Sinai Hospital, Toronto, ON, Canada and ${ }^{3}$ Department of Molecular Genetics, University of Toronto, Toronto, ON, Canada. Correspondence: L Mesbah-Oskui (lia.mesbah.oskui@mail.utoronto.ca)

Received 10 October 2014; revised 2 December 2014; accepted 16 December 2014 
Disc1-L100P CA1 place cells, given their faithful spatial specificity. Importantly, however, the generalized spatial selectivity of Disc1-L100P place cells indicates a reduction in the diversity of simultaneously active place cells. Thus, Disc1-L100P mice may not have the repertoire of active cells required to distinctly code separate locations that are part of a complex or similar spatial memory sequence. The alterations in hippocampal theta power and inhibitory interneuron activity and expression further indicate aberrant hippocampal output in the Disc1-L100P mouse model of schizophrenia. ${ }^{4,19-21}$

\section{MATERIALS AND METHODS}

\section{Animal care}

Place cell and immunohistofluorescence experiments were performed using 3-6-month-old male wild-type (C57BL/6J) and Disc1-L100P mice ( $n=6$ and 3 wild-type and $n=6$ and 3 Disc1-L100P mice for place cell and immunohistofluorescence experiments, respectively) generated as previously described. ${ }^{4}$ Littermates were housed in filtered polycarbonate cages with controlled temperature $\left(21 \pm 1^{\circ} \mathrm{C}\right)$, humidity $(50-60 \%)$, and lighting (on 0700-1900 h). Mice had free access to sterile food and water. All of the procedures were approved by the Toronto Centre for Phenogenomics Animal Care Committee and complied with the Province of Ontario Animals for Research Act 1971 and the Canadian Council on Animal Care requirements. Following surgery, the mice were individually housed.

\section{Surgery}

Microdrive construction and implantation was conducted as previously described. ${ }^{22,23}$ Briefly, a four-tetrode microdrive was positioned above the right CA1 (2.2 mm posterior to Bregma and $2.2 \mathrm{~mm}$ lateral to midline) and the tetrode tips were lowered $800 \mu \mathrm{m}$ below the skull surface. Recordings commenced after a minimum 9-day recovery period.

\section{Habituation and recording procedure}

The mice were habituated to the recording enclosure through daily $30-\mathrm{min}$ exposures for five consecutive days before surgery. Recordings were conducted in a shielded room as mice explored a flat, 49-cm diameter black cylindrical enclosure with a white cue occupying $100^{\circ}$ of arc. Black curtains surrounded the enclosure, blocking distal visual cues. Following recovery, the tetrode tips were lowered in $40 \mu \mathrm{m} /$ day steps. Once putative pyramidal cells and characteristic hippocampal theta rhythm was observed, experimental tetrode position was left fixed and recordings commenced the following day. Recording trials consisted of three 16-min sessions: session 1; $1 \mathrm{~h}$ wait; session 2 with the cue rotated $90^{\circ}$ counterclockwise; a $4 \mathrm{~h}$ wait; and session 3 with the cue back in its original location (Figure 1a). Comparisons of place cell and putative interneuron characteristics were performed using signals collected from session 1.

Signal acquisition and spike sorting

Signal acquisition was performed using a 24-channel Cheetah 32 system (ERP-27, Neuralynx, Bozeman, MT, USA). Signals were amplified 10,000 times and bandpass filtered $(0.6-6 \mathrm{kHz})$. Digitized waveforms $\geqslant 90 \mu \mathrm{V}$ were sampled at $32 \mathrm{kHz}$. Mouse position was collected at $30 \mathrm{~Hz}$ on a grid of $256 \times 256$ pixels, $1.9 \mathrm{~mm}$ per side using light emitting diodes attached to the weight-balanced headstage. Hippocampal local field potential was obtained from the same wires (bandpass filtered $1-475 \mathrm{~Hz}$ with an input range of $\pm 1004 \mu \mathrm{V}$ )

\section{Analysis}

Offline processing was performed using Plexon Offline Sorter to isolate single units (Plexon Instruments, Dallas, TX, USA). Entire units were removed from analysis if they exhibited inter-spike intervals $<2 \mathrm{~ms}$. Single units were classified as either pyramidal neurons or interneurons on the basis of established criteria. ${ }^{24}$ Specifically, putative interneurons were distinguished from pyramidal neurons on the basis of the absence of complex spiking (i.e., bursts of several action potentials occurring 2-10 ms apart), shorter duration action potentials, and higher discharge frequency.

For each pyramidal neuron, a corresponding $25 \times 25$ array of timeaveraged firing rates was generated by dividing, on a pixel-by-pixel basis
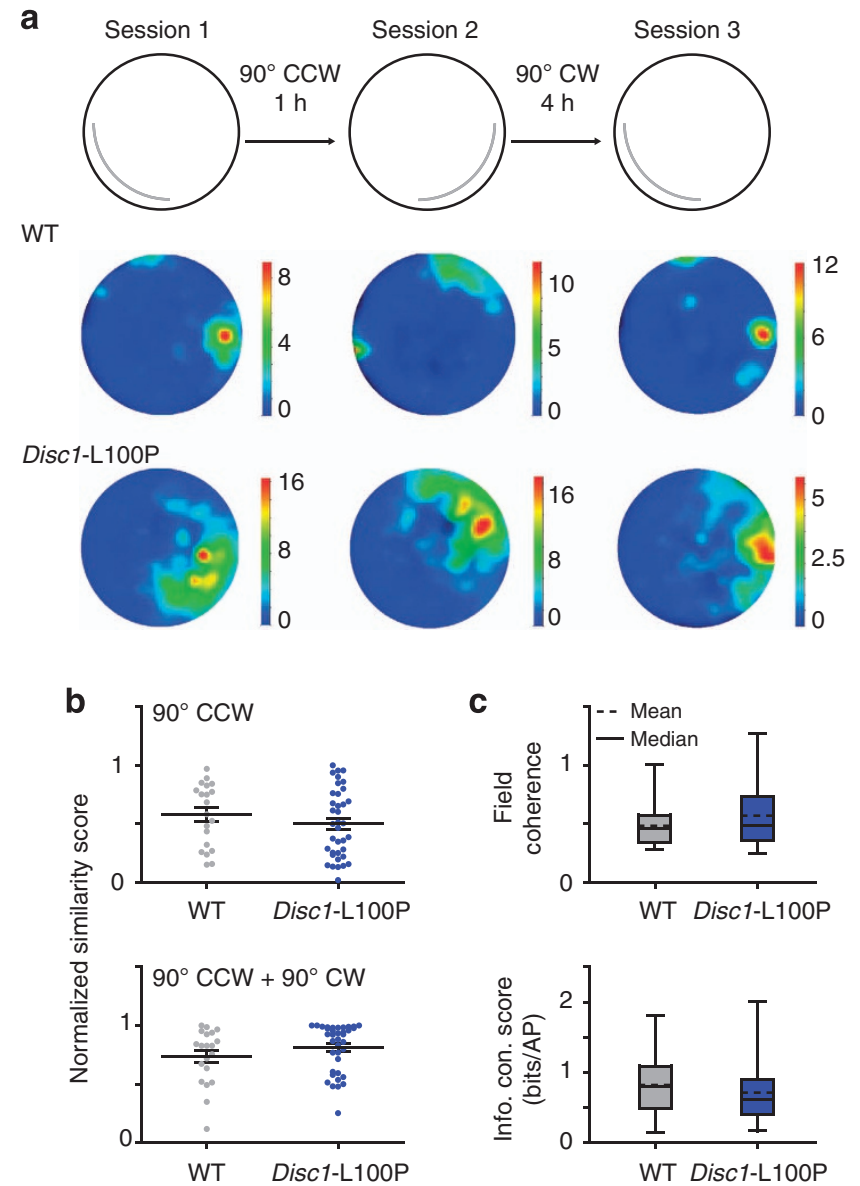

Figure 1. CA1 place cells from mice with a L100P mutation in the disrupted-in-schizophrenia-1 gene (Disc1-L100P) exhibit intact spatial stability. (a) Schematic of cue rotation protocol and exemplar place field rotations from a wild-type (WT) and Disc1-L100P place cell. Color-coded firing rate maps are shown with corresponding frequency scaled to peak (red). (b) Intact coupling of place cell activity to cue between sessions 1 and 2 (upper) and sessions 1 and 3 (lower). Y-axis depicts the similarity score of place fields between sessions 1 and 2 at $270^{\circ}$ (i.e., $90^{\circ}$ counterclockwise upper graph) or sessions 1 and 3 at $0^{\circ}$ (lower graph). N=20 WT and 37 Disc1-L100P place cells. (c) Intact place field coherence and information content scores in Disc1-L100P place cells. Plot whiskers denote minimum and maximum values. The top and bottom of boxes denote the first and third quartile, respectively. Mann-Whitney U-test. $N=80$ WT place cells and 73 Disc1-L100P place cells. CCW, counterclockwise; CW, clockwise; info. con. score, information content score.

$(1.9 \mathrm{~cm} / \mathrm{side})$, the number of spikes by the time in each location. Place fields were defined as a set of $\geqslant 9$ contiguous pixels with a firing rate above the mean firing rate. The spatial specificity of place cell firing was quantified according to the smoothness of place fields formed (spatial coherence), peak and mean in-field firing rates, and information content scores (a measure that reflects the probability of place cell action potential firing relative to spatial location). These well-established parameters of spatial firing quality have been previously described in detail. ${ }^{25}$

Angular rotation quantifies the stability of firing fields between sessions using a pixel-by-pixel cross-correlation between pairs of firing rate arrays. Analysis of angular rotation of place fields was restricted to place cells that exhibited only one field. Correlation values were normalized for each place cell according to the transformation: $x^{\prime}=(x-$ minimum $) /($ maximum - minimum $)$ where $x^{\prime}$ is the normalized value and $x$ is the original value. Comparisons between sessions 1 and 2 were made using the similarity score at $270^{\circ}$ (i.e., $90^{\circ}$ counterclockwise) and comparisons between sessions 1 and 3 were made using the similarity score at $0^{\circ}$. The Freely Moving Animal 
MATLAB Toolbox was used to generate images of place cell firing for use in figures (http://fmatoolbox.sourceforge.net/index.html; MATLAB R12, Mathworks, Natick, MA, USA).

Action potential dynamics were also quantified. The rising slope of action potentials was expressed as the slope of the rising phase of the action potential, when the voltage was halfway to the peak, as a percentage of the peak-to-trough voltage. Similarly, the falling slope of action potentials was expressed as the slope of the falling phase, when the voltage was halfway to the trough, as a percentage of the peak-to-trough voltage.

\section{Verification of tetrode position}

A $50 \mathrm{~mA}$ current was passed through two wires on each tetrode, after which mice were returned to their holding cages for $24 \mathrm{~h}$. Mice were transcardially perfused the next day with ice-cold phosphate buffered saline (PBS) followed by $4 \%$ paraformaldehyde solution. Whole brains were coronally sectioned at $100 \mu \mathrm{m}$ using a vibratome (Pelco Series 1000, Redding, CA, USA). Nissl staining was used to visualize tetrode position (Supplementary Figure S1).

\section{Parvalbumin- and GAD-positive interneuron staining}

The mice were anesthetized with isoflurane and transcardially perfused with ice-cold PBS followed by $4 \%$ paraformaldehyde solution. Whole brains were dissected and immersion fixed overnight in 4\% paraformaldehyde solution. Coronal sections were collected at $100 \mu \mathrm{m}$ using a vibratome (VT1000S, Leica Microsystems, Toronto, ON, Canada) and classified as either rostral ( -1 to $-1.5 \mathrm{~mm}$ from Bregma), central $(-1.5$ to $-2.5 \mathrm{~mm}$, which corresponds to the region sampled for place cell recordings), or caudal $(-2.5$ to $-3.5 \mathrm{~mm})$. Slices were placed in a $4 \%$ bovine serum albumin, $0.1 \%$ Triton-X 100, and PBS solution for $15 \mathrm{~min}$. Rabbit anti-parvalbumin antibody (1:1,000, Swant, PV25, Bellinzona, Switzerland) and/or mouse antiGAD67 (1:200, Millipore, clone 1G10.2, Bedford, MA, USA) was then added and slices were incubated at $4{ }^{\circ} \mathrm{C}$ for $48 \mathrm{~h}$. The sections were washed with the same blocking-permeabilizing solution three times, $10 \mathrm{~min}$ each. Slices were incubated with the appropriate secondary antibody (Cy5-anti-rabbit and/or Cy2-anti-mouse, 1:200; Jackson ImmunoResearch, West Grove, PA, USA) for $2 \mathrm{~h}$ at room temperature and then washed three times, $10 \mathrm{~min}$ each. Slices were held in PBS for $15 \mathrm{~min}$ and mounted on slides with Prolong antifade reagent (Invitrogen, Carlsbad, CA, USA). Sections were imaged under a confocal microscope (Nikon C1si or C2, VC $\times 20 / 0.75$ NA or $\times 4 / 0.13$ lens), the region of interest was defined manually, and automated cell counting was performed (NIS Elements Software, Nikon Canada, Mississauga, ON, Canada). Between 9 and 23 sections were sampled per staining experiment from each of the three mice per genotype.

\section{Statistics}

Comparisons were performed using SigmaStat stastistical software (SPSS, San Jose, CA, USA). The analysis of place cell and putative interneuron characteristics were primarily performed using nonparametric MannWhitney $U$-tests (Supplementary Tables S1 and S2). All other comparisons between Disc1-L100P and wild-type mice were conducted using single(genotype) or two-factor (genotype and hippocampal region) analysis of variance (ANOVA). Differences were considered statistically significant at $P<0.05$.

\section{RESULTS}

CA1 place cell activity was recorded in mice as they explored an open field containing a white polarizing cue that occupied $100^{\circ}$ of arc (Figure 1a). The stability of place cell activity was evaluated through measures that quantified the anchoring of place cell activity (i.e., place fields) to the cue over three separate recording sessions (see Materials and Methods for details; Figure 1a,b). No differences were detected in the anchoring of place cell activity to the cue (sessions 1 vs. 2: $P=0.328$; sessions 1 vs. $3: P=0.163$; Mann-Whitney $U$-test); this analysis of relative place field position was restricted to place cells that exhibited only one place field ( $n=20$ wild-type and 37 Disc1-L100P place cells). These findings identify intact perception of spatial surroundings and faithful encoding of spatial location in Disc1-L100P mice.
We next quantified the spatial specificity of place cell firing. The smoothness of a given place field was evaluated by identifying the correlation between the firing rate in a pixel with the mean firing rate measured in the eight neighboring pixels. Comparisons were conducted on all place cells that were recorded during the first session ( $n=80$ wild-type and 73 Disc1-L100P place cells). The smoothness of Disc1-L100P place fields was similar to wild-type place fields ( $P=0.136$, Mann-Whitney $U$-test; Figure 1c upper). We also did not detect a difference in place cell spatial information content score between wild-type and Disc1-L100P mice (see Materials and Methods; $P=0.076$, Mann-Whitney U-test; Figure 1c lower). These data show that the spatial specificity of CA1 place cells in Disc1-L100P mice is indistinguishable from wild-type mice.

Despite their intact spatial specificity and stability, Disc1-L100P place cells fired more action potentials and exhibited larger place fields relative to wild-type place cells (both $P \leqslant 0.013$, MannWhitney $U$-test; Figure $2 \mathrm{a}-\mathrm{c}$ ). We did not, however, detect any differences in the in-field firing rates between wild-type and Disc1L100P place cells (see Supplementary Information for details, Supplementary Figure $\mathrm{S} 2 \mathrm{~A}, \mathrm{~B}$ ). It is important to note that higher place cell firing rates can be associated with faster speed of movement. ${ }^{26}$ Although Disc1-L100P mice exhibit hyperactivity in a novel environment, analysis of horizontal activity and movement speed showed no differences between Disc1-L100P and wild-type mice, which can be attributed to their habituation to the apparatus (see Materials and Methods and Supplementary Information for details, Supplementary Figure S3A,B). ${ }^{27}$ Disc1L100P place cells did exhibit fewer place fields per cell $(P=0.015$ and $P=0.001$, two-sample Kolmogorov-Smirnov test and Mann-Whitney U-test, respectively; Figure $2 \mathrm{~d}, \mathrm{e})$. Controlling for place field number eliminated differences in firing rate and field size between wild-type and Disc1-L100P place cells, indicating that there are more single-field possessing place cells in the Disc1-L100P mice (Figure 2f).

Disc1-L100P place cell recordings showed a lower signal-tonoise ratio (Figure 3a; $P<0.001$, Mann-Whitney $U$-test). As the measure of noise includes contributions from excitatory and inhibitory neuron action potential signals, we extended our analyses to putative CA1 interneurons (see Materials and Methods for details). We identified a higher number of active putative interneurons in the Disc1-L100P mice (data represented as mean \pm s.e.m.; ratio of putative interneurons to pyramidal neurons: $0.966 \pm 0.337$ in Disc1-L100P mice vs. $0.254 \pm 0.136$ in wild-type mice, $P=0.026$, Kruskal-Wallis one-way ANOVA on ranks). In addition, Disc1-L100P putative interneurons exhibited faster action potential rise kinetics as quantified by the rising slope of their action potentials ( $n=19$ wild-type and 38 Disc1-L100P putative interneurons; $P<0.001$, Mann-Whitney Rank $U$-test), whereas the falling slope was unaffected (Figure $3 b, c)$. Together, these results suggest that, in addition to the gating of inhibitory signaling, the expression and/or activity of the channels mediating the rising phase of action potentials in hippocampal interneurons is altered in Disc1-L100P mice.

Lower hippocampal theta $(5-12 \mathrm{~Hz})$ power was also identified in the Disc1-L100P mice ( $P=0.041$, Kruskal-Wallis one-way ANOVA on ranks; Figure 3d,e). As theta rhythm is mediated by hippocampal inhibitory signaling, we quantified the number of hippocampal inhibitory interneurons by staining for GAD67 and parvalbumin in Disc1-L100P and wild-type mice (Figure 3f). ${ }^{28}$ Despite the increased ratio of inhibitory interneurons to pyramidal neurons that was functionally identified above, Disc1-L100P mice exhibited similar numbers of GAD67-positive hippocampal interneurons $\left(2.1 \pm 0.19 \times 10^{-5}\right.$ cells/ $\mu \mathrm{m}^{2}$ in Disc1-L100P vs. $2.3 \pm 0.20 \times 10^{-5}$ cells $/ \mu \mathrm{m}^{2}$ in wild-type mice; $n=9$ wild-type and 9 Disc1-L100P sections; $P=0.597$, two-way ANOVA; Figure $3 \mathrm{f}, \mathrm{g}$ and Supplementary Figure S4 for a sample series). Alterations, however, in the number of hippocampal parvalbumin-positive 

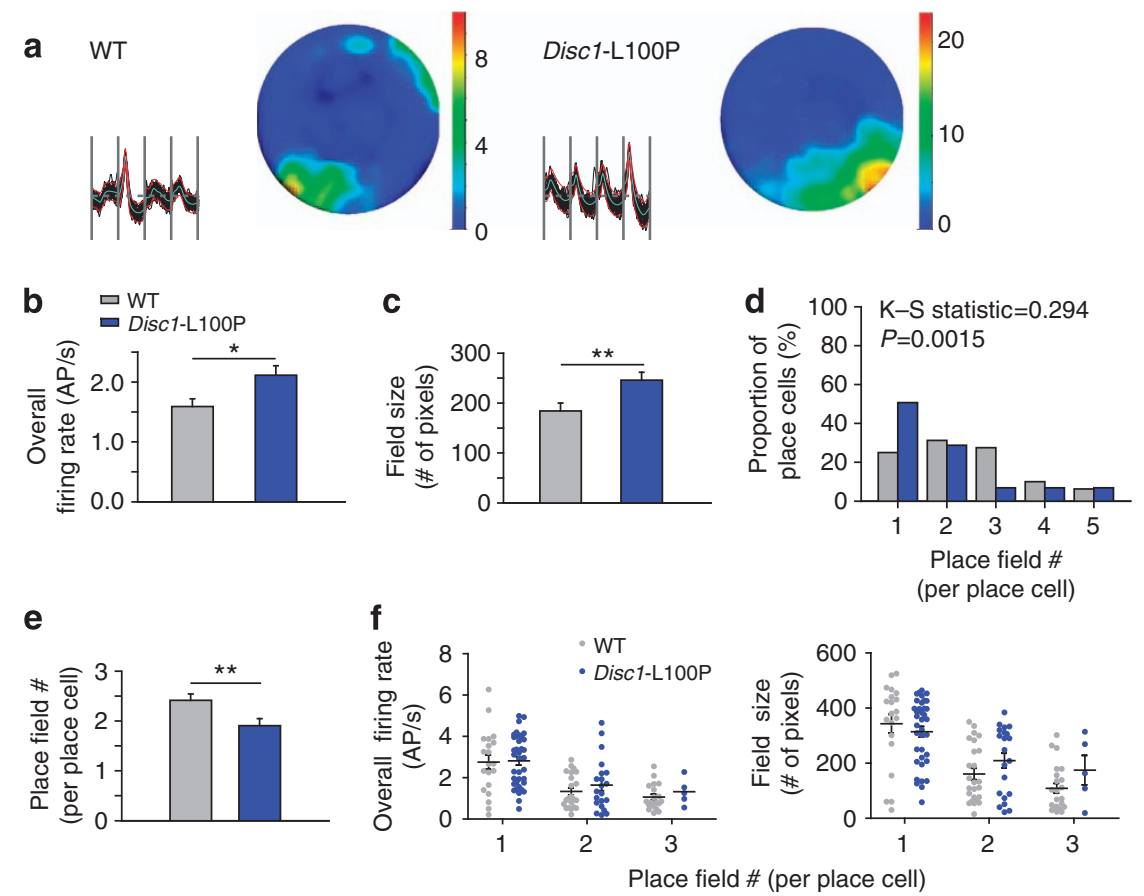

Figure 2. Disc1-L100P mice exhibit fewer place fields per place cell. (a) Tetrode recordings and place fields from a wild-type (WT) and Disc1L100P mouse. Vertical sections on tetrode traces distinguish recordings from individual tetrode wires. Note the difference in the number of fields and their size between the WT and Disc1-L100P place cell. (b) Disc1-L100P place cells show higher overall-firing rates. Data are represented as mean+s.e.m. Mann-Whitney U-test. (c) Disc1-L100P place cells exhibit larger and (d,e) fewer place fields. Two-sample Kolmogorov-Smirnov (K-S) test (d) and Mann-Whitney U-test (e), latter data represented as mean \pm s.e.m. (f) Comparison between place cells displaying equivalent place field numbers identified no differences between Disc1-L100P and WT place cells in average firing rate (left) or field size (right). $N=80$ WT and 73 Disc1-L100P place cells. ${ }^{*} P<0.05,{ }^{* *} P<0.01$. AP, action potential.

interneurons were identified using double-labeling of hippocampal sections (Figure 3f,h). Disc1-L100P mice exhibited fewer parvalbumin-positive interneurons in the central hippocampus, specifically within the $C A 1$, which corresponds to the region sampled for place cell recordings ( $n=23$ wild-type and 22 Disc1-L100P sections; $P=0.011$, two-way ANOVA; post hoc $t$-test for the CA1 $P=0.013$; Figure $3 f, h)$. The deficiency in CA1 parvalbumin-positive interneurons was restricted to the central region of the hippocampus, as we identified increased counts in more rostral sections ( $n=10$ wild-type and 10 Disc1-L100P sections; $P=0.036$, two-way ANOVA) and no difference in counts in more caudal sections ( $n=12$ wild-type and 13 Disc1-L100P sections, Figure $3 \mathrm{~h}$ ). Parvalbumin-positive interneuron redistributions in the cortex and altered numbers in the hippocampus have been previously reported in the Disc1-L100P mice. ${ }^{29}$ Future neurodevelopmental studies of interneuron migration may yield further insight into the functional changes of interneurons and how they affect place cell function. Although decreased numbers of CA1 parvalbumin-positive interneurons may explain the identified deficits in theta rhythm, there could be changes in other interneuron types that contribute to the paradoxical increase in the number of active interneurons identified in the Disc1-L100P mice. ${ }^{28}$

\section{DISCUSSION}

Main findings

Our study of the Disc1-L100P mouse model of schizophrenia identified more single-field-possessing hippocampal place cells, modifications in the numbers and activity of inhibitory interneurons, and lower theta power. The aberrant hippocampal activity in Disc1-L100P mice implicates an impaired ability to distinctly code individual locations, which could contribute to deficiencies in the learning of complex spatial sequences that subserve spatial working memory. ${ }^{15,16}$

Interpretation of findings in relation to previously published work The mechanisms that mediate the number of place fields exhibited by a place cell are not entirely clear. Computational modeling studies suggest that place cell activity is a thresholded sum of grid cell inputs from the medial entorhinal cortex..$^{30-33}$ Importantly, grid cells constitute another major neuronal system that actively encodes spatial location. ${ }^{8}$ Modeling and lesion studies indicate that increased and varied grid cell inputs to place cells promotes more random place field locations with a subsequent increase in the uniqueness of the resulting ensemble place codes. ${ }^{33,34}$ Thus, the increased proportion of single place field-possessing place cells identified in Disc1-L100P mice may relate to alterations in grid cell activity.

Disc1-L100P mice also exhibited reduced hippocampal theta power, which could impair the spatial periodicity of grid cells and may contribute to the observed increase in Disc1-L100P place cells possessing one large field. ${ }^{35}$ Future studies characterizing grid cell spatial periodicity and its association to theta power in Disc1 mice will elucidate the role of reduced theta power and grid cell activity on the spatial selectivity of Disc1-L100P place cells. Importantly, the identified decrease in theta power in Disc1-L100P mice is consistent with studies that have indicated a functional interaction between hippocampal theta rhythm and working memory. ${ }^{36-38}$ Our findings also show that the registration of spatial location is intact in Disc1-L100P mice despite their marked deficits in hippocampal theta power, indicating that the spatial specificity and stability of CA1 place cells is not affected by decreased hippocampal theta rhythm. This is consistent with our previous 
a

口 WT

몯1-L100P

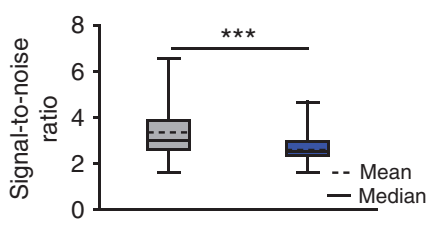

b

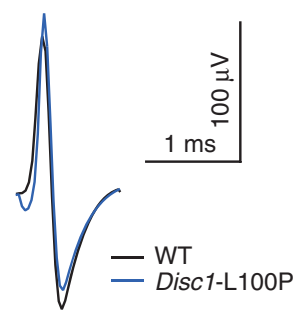

C

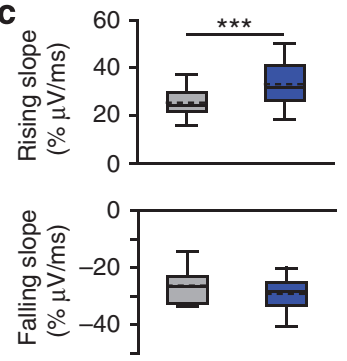

d

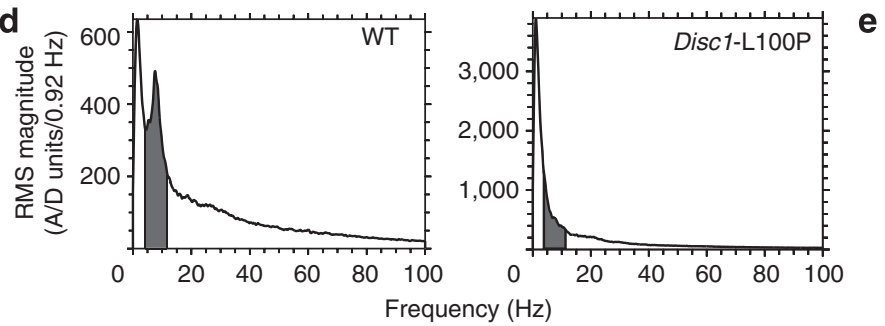

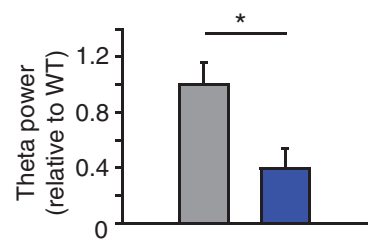
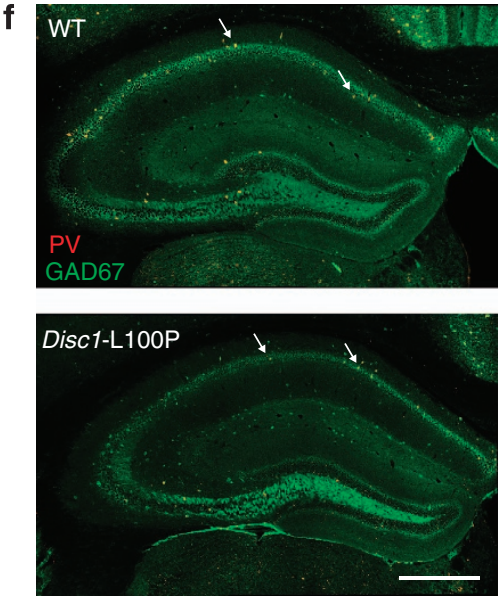

g
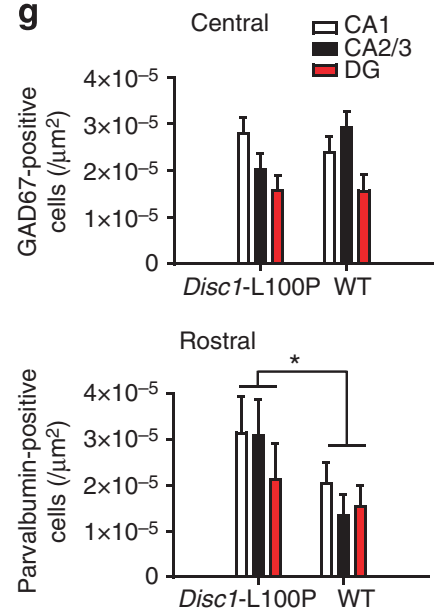

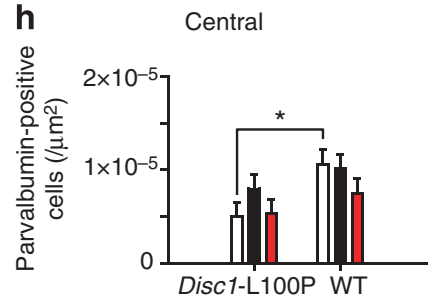

Caudal

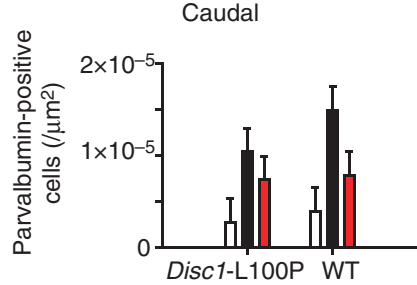

Figure 3. DisC1-L100P mice exhibit aberrant inhibitory interneuron activity and deficits in CA1 parvalbumin-positive interneuron numbers. (a) Disc1-L100P place cells show lower signal-to-noise ratios, when compared with wild-type (WT) place cells, consistent with the elevated numbers of active interneurons recorded from Disc1-L100P mice (see Results for details). Plot whiskers denote minimum and maximum values. The top and bottom of boxes denote the first and third quartile, respectively. Mann-Whitney U-test. N=80 WT and 73 Disc1-L100P place cells. (b) Superimposed action potential traces recorded from a putative interneuron in a WT and a Disc1-L100P mouse. (c) Disc1-L100P putative interneurons exhibit steeper action potential rising slope (upper) and unaffected falling slopes (lower). Mann-Whitney U-test. $N=19 \mathrm{WT}$ and 38 Disc1-L100P putative interneurons. (d) Power spectrums from a WT and Disc1-L100P mouse. Theta band (5-12 Hz) is highlighted in red. (e) Disc1-L100P mice show reduced theta power. Data represented as mean \pm s.e.m. Kruskal-Wallis one-way analysis of variance (ANOVA) on ranks. $N=6$ WT and 6 Disc1-L100P recordings. (f) Example confocal images of anti-parvalbumin and anti-GAD67 fluorescence from a WT (upper) and Disc1-L100P (lower) central hippocampal section. Arrows point to some of the cells that stained positive for both parvalbumin and GAD67. Bar $=500 \mu \mathrm{m}$. (g) Disc1-L100P mice possess similar numbers of GAD67-positive cells in the central hippocampus. Plotted data show mean \pm s.e.m. two-way ANOVA. N = 3 WT and 3 Disc1-L100P mice. (h) Disc1-L100P mice exhibit alterations in parvalbumin-positive interneuron numbers. Relative to WT mice, Disc1-L100P mice exhibit fewer parvalbumin-positive interneurons in the central CA1 (upper), more parvalbumin-positive interneurons in the rostral hippocampus (lower left), and similar levels of parvalbumin-positive interneurons in the caudal hippocampus (lower right). Plotted data show mean \pm s.e.m. two-way ANOVA. Data represents average of 9 to 23 sections from $N=3$ WT and 3 Disc1-L100P mice. ${ }^{* P}<0.05,{ }^{* *} P<0.001$. A/D, analog-to-digital; Disc1-L100P, disrupted-in-schizophrenia-1 gene; RMS, root mean square.

findings showing intact spatial navigation in Disc1-L100P mice in the presence of navigational cues. ${ }^{4}$

The alterations in CA1 inhibitory interneuron activity and parvalbumin-positive interneuron numbers are consistent with reports identifying aberrant GABAergic systems in schizophrenic patients and animal models with schizophrenia-like endophenotypes. ${ }^{17,19,39}$ Proper functioning of CA1 parvalbuminpositive interneurons is required for faithful spatial working memory in mice. ${ }^{20}$ Future studies, designed to identify the mechanisms leading to altered hippocampal parvalbumin-positive interneuron distribution and how this relates to spatial working memory, may aid in our understanding of the molecular and cellular mechanisms contributing to cognitive dysfunction in schizophrenia.

The increased proportion of active interneurons and lower number of parvalbumin-positive interneurons in the central hippocampus of Disc1-L100P mice suggest that reciprocal inhibitory connections may be impaired in this region. Importantly, interneuron-specific interneurons have been identified in 
the CA1 and can strongly regulate the firing rate and timing of oriens-lacunosum moleculare interneurons, which in turn, could regulate place cell burst firing. ${ }^{40,41}$ Future studies on Disc1 interneuron alterations, including redistributions, are required to determine their contribution to the intra-hippocampal mechanisms that enhance single-field firing in Disc1-L100P place cells.

This study is the first to identify an alteration in the number of place fields expressed by place cells in mice that exhibit a schizophrenia-like endophenotype. To our knowledge, there is only one other study that has examined place cell activity in mice that exhibit schizophrenia-like behavior, which included deficits in spatial working memory. Specifically, mice with a forebrainspecific knockout of calcineurin possess place cells with intact spatial specificity, consistent with our findings in the Disc1-L100P mice. $^{42}$ In addition, calcineurin knockout mice exhibited impaired rest-related neural information processing, as indexed by the 'replay' of place cell activity that was observed during prior spatial exploration. Place cell activity has also been characterized in rats following induction of reversible deficits in spatial working memory, elicited by the administration of a cannabinoid receptor agonist. ${ }^{43}$ This study similarly reported no alterations in the spatial specificity of CA1 place cells during working memory impairment. Disruption of theta oscillations and theta-coordinated place cell activity, however, was reported, which is also consistent with our findings. No alterations in place field size or number were reported in the above studies. Importantly, both of these studies examined place cell activity while animals traversed a linear track/ maze as opposed to an open field. Thus, the area explored in these experiments was restricted, which would decrease the probability of observing multiple place fields per place cell and make identifying alterations in place field number difficult. Finally, our results differ from those reported in mice that carried a selective knockout of $\mathrm{N}$-methyl D-aspartate (NMDA) receptors on parvalbumin-positive interneurons. ${ }^{44}$ Specifically, these mice possessed place cells with impaired spatial specificity but normal place field numbers and size. Importantly, these mice did not exhibit alterations in behaviors associated with schizophrenia.

The generalization of spatial selectivity in Disc1-L100P place cells suggests that the generated ensemble place codes are not as unique as those of wild-type mice. Faithful working memory requires sequence learning, and multiple-item recall models postulate that activated cell ensembles act to code for individual sequence elements. ${ }^{11,16}$ Moreover, each ensemble is distinguished from the others through its temporal coupling to theta oscillations. ${ }^{16}$ In the context of these models, deficits in theta power and decreased uniqueness of ensemble place codes indicate impaired sequence learning in Disc1-L100P mice, which is consistent with their deficits in spatial working memory. ${ }^{4}$

There is accumulating evidence that disturbances in neural oscillations can act as an effective endophenotype for schizophrenia. ${ }^{45}$ Our findings provide further insight on the potential pathophysiological processes that could give rise to decreased theta amplitude, i.e., alterations in hippocampal interneuron signaling and numbers. Importantly, impaired resting and evoked theta oscillations during sensory gating in patients with schizophrenia and first-degree relatives have been reported, with these impairments exhibiting a high degree of heritability. ${ }^{46}$ Moreover, our findings suggest aberrant hippocampal ensemble coding in Disc1-L100P mice, which provides a potential mechanistic explanation for the deficits in intrinsic sequence learning and working memory identified in patients with schizophrenia. ${ }^{47}$

\section{Strengths and limitations of this study}

This study characterizes hippocampal neuronal activity in freely behaving mice that contain a mutation in a gene that has been associated with schizophrenia and affective disorder. ${ }^{12-14}$ The findings of this study have important implications regarding the neuropathology of cognitive dysfunction in schizophrenia, indicating simplified coding as a contributor to impaired working memory. Modeling schizophrenia in animals is debated as there is no ubiquitous biological hallmark of the disorder. ${ }^{48}$ Nonetheless, Disc1-L100P mice exhibit deficits in information processing, spatial working memory, and brain volume that are consistent with findings in patients with schizophrenia. ${ }^{4,12}$ Moreover, antipsychotics alleviate the deficits in information processing in Disc1-L100P mice. ${ }^{4}$ These findings support the use of Disc1-L100P mice to model a schizophrenia-like disorder. In addition, neurons with properties similar to place cells and grid cells have been identified in humans, further indicating that the findings of this study extend to patients with schizophrenia. ${ }^{49,50}$ Finally, this study describes alterations in the encoding of spatial location by place cells that can potentially explain the defects in spatial working memory that were previously detected in Disc1-L100P mice.

Implications for future research, policy, and practice

Determining the mechanism(s) that elicit generalized place cell selectivity and aberrant CA1 inhibitory signaling in Disc1-L100P mice will increase our understanding of the pathophysiological processes contributing to cognitive dysfunction in these mice. Such an understanding may facilitate the identification of therapeutic targets for mediating cognitive dysfunction in schizophrenia and other disorders, of which none currently exist.

\section{Conclusions}

This study identifies alterations in the neuronal coding of environmental information in mice with a schizophrenia-like endophenotype and implicates simplified coding as a contributor to deficient working memory in schizophrenia. This study also provides support for aberrant GABAergic signaling in schizophrenia.

\section{ACKNOWLEDGMENTS}

This work is dedicated to the memory of Robert U. Muller. The authors thank Jeremy Barry for his technical assistance with place cell recordings. The authors thank Albert Wong and Frankie Lee for feedback and providing Sigma anti-parvalbumin antibody. The authors also thank Sabine Cordes for use of her laboratory vibratome.

\section{CONTRIBUTIONS}

LM-O and JG performed the experiments and analyzed the data. LM-O, JG, and JCR wrote the paper and designed the study.

\section{COMPETING INTERESTS}

The authors declare no conflict of interest.

\section{FUNDING}

This work was supported by the Canadian Institute of Health Research (CIHR MOP \#147865 and 111198). Equipment used for these experiments were obtained through a CFI/ORF grant to JCR (\#20646). JCR was supported by a Tier 1 Canada Research Chair in Learning and Memory.

\section{REFERENCES}

1 Keefe RS, Roitman SE, Harvey PD, Blum CS, DuPre RL, Prieto DM et al. A pen-andpaper human analogue of a monkey prefrontal cortex activation task: spatial working memory in patients with schizophrenia. Schizophr Res 1995; 17: 25-33.

2 Park S, Holzman PS. Schizophrenics show spatial working memory deficits. Arch Gen Psychiatry 1992; 49: 975-982.

3 Park S, Puschel J, Sauter BH, Rentsch M, Hell D. Spatial working memory deficits and clinical symptoms in schizophrenia: a 4-month follow-up study. Biol Psychiatry 1999; 46: 392-400

4 Clapcote SJ, Lipina TV, Millar JK, Mackie S, Christie S, Ogawa F et al. Behavioral phenotypes of Disc1 missense mutations in mice. Neuron 2007; 54: 387-402. 
5 Kvajo M, McKellar H, Arguello PA, Drew LJ, Moore $H$, MacDermott $A B$ et al. A mutation in mouse Disc1 that models a schizophrenia risk allele leads to specific alterations in neuronal architecture and cognition. Proc Natl Acad Sci USA 2008; 105: 7076-7081.

6 Koike H, Arguello PA, Kvajo M, Karayiorgou M, Gogos JA. Disc1 is mutated in the 129S6/SvEv strain and modulates working memory in mice. Proc Natl Acad Sci USA 2006; 103: 3693-3697.

7 Hikida T, Jaaro-Peled H, Seshadri S, Oishi K, Hookway C, Kong S et al. Dominantnegative DISC1 transgenic mice display schizophrenia-associated phenotypes detected by measures translatable to humans. Proc Natl Acad Sci USA 2007; 104: 14501-14506.

8 Derdikman D, Moser El. A manifold of spatial maps in the brain. Trends Cogn Sci 2010; 14: 561-569.

9 Sevy S, Davidson M. The cost of cognitive impairment in schizophrenia. Schizophr Res 1995; 17: 1-3.

10 Meltzer HY, Park S, Kessler R. Cognition, schizophrenia, and the atypical antipsychotic drugs. Proc Natl Acad Sci USA 1999; 96: 13591-13593.

11 Baddeley A. Working memory: looking back and looking forward. Nat Rev Neurosci 2003; 4: 829-839.

12 Blackwood DH, Fordyce A, Walker MT, St Clair DM, Porteous DJ, Muir WJ. Schizophrenia and affective disorders--cosegregation with a translocation at chromosome 1q42 that directly disrupts brain-expressed genes: clinical and P300 findings in a family. Am J Hum Genet 2001; 69: 428-433.

13 Brandon NJ, Sawa A. Linking neurodevelopmental and synaptic theories of mental illness through DISC1. Nat Rev Neurosci 2011; 12: 707-722.

14 Duff BJ, Macritchie KA, Moorhead TW, Lawrie SM, Blackwood DH. Human brain imaging studies of DISC1 in schizophrenia, bipolar disorder and depression: a systematic review. Schizophr Res 2013; 147: 1-13.

15 Fenton AA, Kao HY, Neymotin SA, Olypher A, Vayntrub Y, Lytton WW et al. Unmasking the CA1 ensemble place code by exposures to small and large environments: more place cells and multiple, irregularly arranged, and expanded place fields in the larger space. J Neurosci 2008; 28: 11250-11262.

16 Lisman JE, Jensen O. The theta-gamma neural code. Neuron 2013; 77: 1002-1016.

17 Lewis DA, Hashimoto T, Volk DW. Cortical inhibitory neurons and schizophrenia. Nat Rev Neurosci 2005; 6: 312-324.

18 Uhlhaas PJ, Singer W. Abnormal neural oscillations and synchrony in schizophrenia. Nat Rev Neurosci 2010; 11: 100-113.

19 Lodge DJ, Behrens MM, Grace AA. A loss of parvalbumin-containing interneurons is associated with diminished oscillatory activity in an animal model of schizophrenia. J Neurosci 2009; 29: 2344-2354.

20 Murray AJ, Sauer JF, Riedel G, McClure C, Ansel L, Cheyne L et al. Parvalbuminpositive CA1 interneurons are required for spatial working but not for reference memory. Nat Neurosci 2011; 14: 297-299.

21 Jones MW, Wilson MA. Theta rhythms coordinate hippocampal-prefrontal interactions in a spatial memory task. PLoS Biol 2005; 3: e402.

22 Taverna FA, Georgiou J, McDonald RJ, Hong NS, Kraev A, Salter MW et al. Defective place cell activity in nociceptin receptor knockout mice with elevated NMDA receptor-dependent long-term potentiation. J Physiol 2005; 565: 579-591.

23 Yan J, Zhang Y, Jia Z, Taverna FA, McDonald RJ, Muller RU et al. Place-cell impairment in glutamate receptor 2 mutant mice. J Neurosci 2002; 22: RC204.

24 Ranck JB Jr. Studies on single neurons in dorsal hippocampal formation and septum in unrestrained rats. I. Behavioral correlates and firing repertoires. Exp Neurol 1973; 41: 461-531.

25 Kubie JL, Muller RU, Bostock E. Spatial firing properties of hippocampal theta cells. J Neurosci 1990; 10: 1110-1123.

26 Geisler C, Robbe D, Zugaro M, Sirota A, Buzsaki G. Hippocampal place cell assemblies are speed-controlled oscillators. Proc Natl Acad Sci USA 2007; 104: 8149-8154.

27 Lipina TV, Niwa M, Jaaro-Peled H, Fletcher PJ, Seeman P, Sawa A et al. Enhanced dopamine function in DISC1-L100P mutant mice: implications for schizophrenia. Genes Brain Behav 2010; 9: 777-789.

28 Wulff $\mathrm{P}$, Ponomarenko AA, Bartos M, Korotkova TM, Fuchs EC, Bahner F et al. Hippocampal theta rhythm and its coupling with gamma oscillations require fast inhibition onto parvalbumin-positive interneurons. Proc Natl Acad Sci USA 2009; 106: $3561-3566$
29 Lee FH, Zai CC, Cordes SP, Roder JC, Wong AH. Abnormal interneuron development in disrupted-in-schizophrenia-1 L100P mutant mice. Mol Brain 2013; 6: 20.

30 McNaughton BL, Battaglia FP, Jensen O, Moser El, Moser MB. Path integration and the neural basis of the 'cognitive map'. Nat Rev Neurosci 2006; 7: 663-678.

31 Solstad T, Moser El, Einevoll GT. From grid cells to place cells: a mathematical model. Hippocampus 2006; 16: 1026-1031.

32 Franzius M, Vollgraf R, Wiskott L. From grids to places. J Comput Neurosci 2007; 22: 297-299.

33 Fuhs MC, Touretzky DS. A spin glass model of path integration in rat medial entorhinal cortex. J Neurosci 2006; 26: 4266-4276.

34 Van Cauter T, Poucet B, Save E. Unstable CA1 place cell representation in rats with entorhinal cortex lesions. Eur J Neurosci 2008; 27: 1933-1946.

35 Brandon MP, Bogaard AR, Libby CP, Connerney MA, Gupta K, Hasselmo ME. Reduction of theta rhythm dissociates grid cell spatial periodicity from directional tuning. Science 2011; 332: 595-599.

36 Hasselmo ME. What is the function of hippocampal theta rhythm? - Linking behavioral data to phasic properties of field potential and unit recording data. Hippocampus 2005; 15: 936-949.

37 Carr MF, Jadhav SP, Frank LM. Hippocampal replay in the awake state: a potential substrate for memory consolidation and retrieval. Nat Neurosci 2011; 14: 147-153.

38 Mehta MR, Lee AK, Wilson MA. Role of experience and oscillations in transforming a rate code into a temporal code. Nature 2002; 417: 741-746.

39 Zhang ZJ, Reynolds GP. A selective decrease in the relative density of parvalbumin-immunoreactive neurons in the hippocampus in schizophrenia. Schizophr Res 2002; 55: 1-10

40 Tyan L, Chamberland S, Magnin E, Camire O, Francavilla R, David LS et al. Dendritic inhibition provided by interneuron-specific cells controls the firing rate and timing of the hippocampal feedback inhibitory circuitry. J Neurosci 2014; 34: 4534-4547.

41 Klausberger T, Magill PJ, Marton LF, Roberts JD, Cobden PM, Buzsaki G et al. Brainstate- and cell-type-specific firing of hippocampal interneurons in vivo. Nature 2003; 421: 844-848.

42 Suh J, Foster DJ, Davoudi H, Wilson MA, Tonegawa S. Impaired hippocampal ripple-associated replay in a mouse model of schizophrenia. Neuron 2013; 80: 484-493.

43 Robbe D, Buzsaki G. Alteration of theta timescale dynamics of hippocampal place cells by a cannabinoid is associated with memory impairment. J Neurosci 2009; 29: 12597-12605.

44 Korotkova T, Fuchs EC, Ponomarenko A, von Engelhardt J, Monyer H. NMDA receptor ablation on parvalbumin-positive interneurons impairs hippocampal synchrony, spatial representations, and working memory. Neuron 2010; 68: 557-569.

45 Uhlhaas PJ, Singer W. Neuronal dynamics and neuropsychiatric disorders: toward a translational paradigm for dysfunctional large-scale networks. Neuron 2012; 75: 963-980.

46 Hong LE, Summerfelt A, Mitchell BD, McMahon RP, Wonodi I, Buchanan RW et al. Sensory gating endophenotype based on its neural oscillatory pattern and heritability estimate. Arch Gen Psychiatry 2008; 65: 1008-1016.

47 Siegert RJ, Weatherall $M$, Bell EM. Is implicit sequence learning impaired in schizophrenia? A meta-analysis. Brain Cogn 2008; 67: 351-359.

48 Lipska BK, Weinberger DR. To model a psychiatric disorder in animals: schizophrenia as a reality test. Neuropsychopharmacology 2000; 23: 223-239.

49 Ekstrom AD, Kahana MJ, Caplan JB, Fields TA, Isham EA, Newman EL et al. Cellular networks underlying human spatial navigation. Nature 2003; 425: 184-188.

50 Doeller CF, Barry C, Burgess N. Evidence for grid cells in a human memory network. Nature 2010; 463: 657-661.

(i) $(-)$ This work is licensed under a Creative Commons Attribution(the images or other third party material in this article are included in the article's Creative Commons license, unless indicated otherwise in the credit line; if the material is not included under the Creative Commons license, users will need to obtain permission from the license holder to reproduce the material. To view a copy of this license, visit http:// creativecommons.org/licenses/by-nc-nd/4.0/

Supplementary Information accompanies the paper on the npj Schizophrenia website (http://www.nature.com/npjschz) 